\title{
Ampleur et facteurs de risque de l'obésité des enfants à Bamako
}

\author{
Magnitude and risk factors of obesity among children Bamako
}

Fofana A.S. ${ }^{1 *}$, Sidibé F.D. ${ }^{2}$, Fofana B.S. ${ }^{1}$, Traoré ${ }^{1}{ }^{1}$

\begin{abstract}
${ }^{1}$ Laboratoire National de la Santé, Bamako Mali,
2 Institut de Recherche en Santé Publique

*Auteur de correspondance : aminataf@yahoo.fr

Résumé
\end{abstract}

Introduction : Au Mali, il n'existe aucune information sur l'état nutritionnel des écoliers. Les données de cette étude serviront de référence. Méthode : Nous avons mené une étude descriptive par sondage aléatoire dans six écoles du district de Bamako de mars à mai 2015. Un total de 92 enfants âgés de 8 à 11 ans a été recruté. L'IMC était déterminé par anthropométrie. La composition corporelle a été évaluée par la technique de dilution isotopique. Résultats : Par la classification de I'OMS (l'IMC/âge) $11,9 \%$ des enfants en surpoids et $6 \%$ obèses ( $1,78 \%$ pour les garçons et $4,22 \%$ pour les filles). Par la composition corporelle, le taux d'obésité était de $26 \%$ (pour les garçons $\mathrm{FM}>25 \%$ : $11,9 \%$ et pour les filles $\mathrm{FM}>$ $30 \%: 14,1 \%$ ). Le taux d'hypertension artérielle était de $12 \%$. Seulement $16,2 \%$ et $15,8 \%$ des enfants avaient respectivement une bonne connaissance des pratiques nutritionnelles et une bonne estime de soi. Parmi les enfants obèses, $88,9 \%$ étaient des sédentaires, $45,8 \%$ étaient hypertendus, $70 \%$ avaient de mauvais point de vue nutritionnel, $87,5 \%$ avaient une mauvaise estime de soi. Les résultats montrent que l'hypertension artérielle et l'estime de soi avaient une relation statistique avec l'obésité. Conclusion : cette étude nous a permis de constater que les mesures anthropométriques sousestiment l'obésité. II est important de mener d'autres études pour mieux comprendre l'étiologie de l'obésité afin que des mesures soient axées sa prévention.

Mots cles : obésité, composition corporelle, masse grasse, enfants, Mali

Abstract

Introduction: In Mali no information exists on the nutritional status of primary school children. Data of this study will be reference. Method: We conducted a randomized descriptive randomized study in six schools of Bamako from March to May 2015. A total of 92 children aged 8-11 years from primary schools were recruited. BMI was determining by anthropometry. Body composition was derived from isotope dilution technique. Results: By WHO classification, (BMI-for-age), $11.9 \%$ of children were overweight, $6 \%$ obese. According body composition rate of obesity was $26 \%$, rate of high blood pressure was of $12 \%$. Only $16.2 \%$ and $15.8 \%$ of children had respectively a good knowledge of practices in nutrition and body self-esteem.

Among obese children, $88.9 \%$ were sedentary, $45.8 \%$ were hypertensive, $70 \%$ had bad nutritional practices, $87.5 \%$ bad self-estimate. However, among risk factors analyzed, our results showed that statistical relationship was found between child hypertensive and obesity and body self-esteem. Conclusion: this study allowed us to see that anthropometric measures underact obesity. It important to conduct others studies to understand the etiology of obesity so that measures should focus on it prevention.

Key words: obesity, body composition, fat mass, children, Mali

Introduction

Le surpoids et l'obésité se définissent comme une accumulation anormale ou excessive de graisse corporelle qui peut nuire à la santé (OMS). Ils constituent un problème majeur de santé publique en raison de leur retentissement potentiel sur la santé et de leur fréquence croissante. Le surpoids est un déterminant important de la santé qui expose les personnes concernées à de nombreuses pathologies cardiovasculaires, métaboliques, articulaires, vésiculaires et cancéreuses, ainsi qu'à une augmentation de la mortalité. L'obésité chez les enfants présente en outre un risque important de persistance à l'âge adulte. Les enfants obèses deviennent des adultes obèses dans des proportions qui varient selon les études, de 20 à $50 \%$ si l'obésité était présente avant la puberté et de 50 à $70 \%$ après la puberté [1-2].

L'Organisation Mondiale de la Santé (OMS) estime qu'en 2014 plus de 1,9 milliards d'adultes (personne de 18 ans et plus) étaient en surpoids et plus de 600 millions étaient obèses [3]. Globalement $13 \%$ de la population mondiale adulte étaient obèses et $39 \%$ en surpoids en 2014 [3]. Le surpoids et l'obésité concernaient près de 42 millions d'enfants de moins de 5 ans et plus de 340 millions d'enfants et adolescents âgés de 5 à 19 ans étaient en surpoids ou obèses en 2016 [3]. La prévalence de l'obésité a presque triplé au niveau mondial entre 1975 et 2014 , et l'OMS prédit que ce taux passera respectivement à $41 \%$ pour les femmes et $30 \%$ pour les hommes d'ici 10 ans [3]. Autrefois considérés comme des problèmes spécifiques des pays à haut revenu, le surpoids et l'obésité sont désormais en augmentation dans les pays à revenu faible et intermédiaire, en particulier en milieu urbain [4].

En Afrique, les maladies non transmissibles (MNT) représentent $28 \%$ du taux de morbidité et $35 \%$ de la mortalité. Ce taux devrait grimper entre $60 \%$ et $65 \%$ d'ici 2020 [5]. L'Institut de cardiologie ougandais prévoit que les cardiopathies liées à l'obésité seront la principale cause de décès en Afrique subsaharienne d'ici à 2020 [6]. Dans des pays comme le Sénégal, l'Ouganda et le Kenya, l'obésité est en augmentation bien que la malnutrition continue de constituer le plus gros problème. En outre, au Kenya $22 \%$ des enfants âgés de 3 à 5 ans 
(2003) sont en surpoids ou obèses. Au Sénégal, la prévalence de l'obésité infantile était de 9,34 \% [7]. L'Afrique subsaharienne n'est pas encore en situation d'alerte devant ce phénomène, cependant les données sont encore rares.

Au Mali, les principales MNT sont en passe de devenir le principal fardeau épidémiologique avec $7 \%$ pour l'obésité, $3.3 \%$ pour le diabète et $15 \%$ pour l'hypertension. L'OMS indique que le taux de prévalence de l'obésité chez les adultes, estimé à $4,8 \%$ en 2008 , est passé à 10,5\% en 2013 [8]. II n'existe pas de données sur l'obésité infantile au Mali, mais ils ne sont pas à l'abri du fléau d'où la nécessité de faire une évaluation précise de la situation actuelle.

La réduction de la prévalence de l'obésité est l'un des enjeux majeurs du 21ème siècle. Les pays en voie de développement (PVD) ont l'opportunité de tirer des leçons de ce qui s'est passé dans les pays développés pour profiter du développement en évitant ses effets néfastes. Leur nouveau défi est de freiner la progression du surpoids au fur et à mesure du développement socioéconomique, de l'urbanisation et de la diminution des malnutritions par carences.

Dans cette étude, nous ciblons les enfants scolarisés car il est plus facile d'introduire des interventions au niveau scolaire et le plus souvent, les habitudes apprises à l'école continuent plus tard dans la vie. L'étude avait pour but d'évaluer le statut nutritionnel des enfants. Le niveau de l'obésité sera déterminé à l'aide de la mesure de l'indice de masse corporelle (IMC/A Z-score) et de la composition corporelle. L'IMC étant l'indicateur de corpulence couramment utilisé pour évaluer l'obésité, mais vue son incapacité à distinguer la masse grasse de la masse musculaire il est insuffisant pour déterminer exactement l'état de santé d'un individu. La composition corporelle, se basant sur les compartiments est une variante importante de la santé et permet de mieux appréhender l'état nutritionnel et de santé d'un individu [3]. La technique de dilution isotopique appliquée pour l'évaluation de la composition corporelle est une approche sûre, précise et relativement non invasive.

\footnotetext{
Méthodes

L'étude a été réalisée dans six (6) écoles dont trois publiques et privées des six communes du District de Bamako. Elle a concerné 92 enfants scolarisés en bonne santé, de 8 et 11 ans en classe de 3ème à la 6ème année du premier cycle de l'enseignement fondamental. La période de collecte de données s'est étalée sur trois mois, allant de mars à mai 2015 et l'étude a duré trois (2014-2016).

La sélection des écoles a été faite à partir des listes d'établissements fournies par les Directions de Centres d'Animation Pédagogique (DCAP) du Ministère de l'enseignement général. Le recrutement des élèves participants a été fait au niveau des écoles retenues, à partir des listes des élèves âgés de 8 à 11 ans du premier cycle fondamental par échantillonnage aléatoire, et à partir du logiciel ENA SMART. Les consentements
}

éclairés des enfants et de leurs parents ont été obtenus. La proposition a été passée au comité d'éthique de I'Institut National de recherche en Santé publique (INRSP).

Le poids, la taille et le tour de taille des élèves ont été mesurés et comparés à la norme de l'OMS. Des renseignements individuels et groupés sur la stratégie d'une alimentation saine ont été recueillis auprès des élèves. Les différents prélèvements et mesures ont été effectués dans les écoles par l'équipe de recherche. Le poids de l'élève habillé légèrement, pied nu a été mesuré à $0.1 \mathrm{Kg}$ près en utilisant une balance électronique pour adulte placée sur une surface plane équilibrée. Une deuxième mesure a été faite et enregistrée. Une troisième mesure a été faite si les deux premières mesures sont différentes de $0.5 \mathrm{Kg}$. La précision de la balance devrait être vérifiée périodiquement à l'aide d'une masse étalon connue. La taille de l'élève a été mesurée à $0,1 \mathrm{Cm}$ près, à l'aide d'une toise fixe. Le tour de taille de l'élève a été mesuré à $0,1 \mathrm{~cm}$ près à l'aide d'un ruban mètre. Cette mesure est faite à la fin d'une expiration normale avec les bras relâchés de chaque côté du corps, en suivant la ligne axillaire, au point situé à mi-distance inférieure de la dernière côte et la crête iliaque.

Les mesures (Poids, taille, tour de taille, date de naissance, sexe....) sont saisies dans logiciel Anthroplus de l'OMS. Ce logiciel permet de calculer l'IMC-âge, de classer les enfants âgés de 5 à 19 ans selon leur état nutritionnel et de surveiller leur croissance. Les seuils du Z-score IMC-âge sont les suivants: sévèrement émacié $(<-3)$, émacié $(>-3$ et $<-2)$, Normal $(>-1$ et $<1)$, surpoids $(>+1$ et $<+2)$, Obésité $(>+2)$.

La composition corporelle : le poids corporel est un indicateur relativement pauvre de l'état de santé et du statut nutritionnel. Un indicateur plus important est la composition corporelle, qui permet de savoir de quoi le poids corporel d'un individu est constitué. Le corps humain est souvent subdivisé en deux compartiments : masse grasse (MG) et masse maigre (MM). Ceci est connu comme le modèle à deux compartiments. Le deutérium est un isotope stable (non-radioactif) d'hydrogène, ayant comme symbole ${ }^{2} \mathrm{H}$ ou $\mathrm{D}$. II est utilisé comme un marqueur. Pour cela, des échantillons de salive des écoliers ont été prélevés 30 minutes au moins, après leur dernier repas ou boisson. Afin de prévoir les répétitions d'analyses, on prélève $4 \mathrm{ml}$ de salive. Puis, le participant ingère oralement une dose d'oxyde de deutérium $\left({ }^{2} \mathrm{H}_{2} \mathrm{O}\right.$ ou $\left.\mathrm{D}_{2} \mathrm{O}\right)$ de $0,5 \mathrm{~g} / \mathrm{Kg}$ de poids exactement pesées à $0.01 \mathrm{~g}$ près. La quantité de la dose de deutérium ingéré et l'heure de l'ingestion sont notées. Le deutérium est mélangé avec toute l'eau corporelle totale et l'équilibre est atteint 3 heures après son ingestion. C'est alors qu'un premier échantillon de salive post-dose sera recueilli, puis un second à la 4 ème heure dans des tubes clairement étiquetés. L'élève doit donc rester à jeun pendant 4 heures. L'oxyde de deutérium est métabolisé dans le corps de la même façon que l'eau, et il est dispersé à travers l'eau corporelle en quelques 
heures. Tout le deutérium sera éliminé du corps de l'enfant à travers sa salive, son urine et sa sueur. Les échantillons seront stockés à $-28^{\circ} \mathrm{C}$ avant leur analyse. Le dosage de l'enrichissement au deutérium est fait à l'aide d'une photométrie infrarouge à transformer de Fourier (FTIR). Les seuils d'obésité selon la composition corporelle en $M G$ sont : chez les garçons ( $M G \geq 25 \%$ ) et chez les filles $M G \geq 30 \%$ ).

L'activité physique a été mesurée à l'aide d'un accéléromètre de type «Actigraph Wgt3x-BT ». L'avantage est qu'il ne modifie pas les habitudes quotidiennes du sujet. Initialisé, l'élève le porte autour de la taille. II place l'Actigraph sur les os du tour de hanche de sorte qu'il repose directement sous l'aisselle droite. Les informations sont enregistrées toutes les minutes pendant sept jours pour évaluer l'intensité de l'activité physique et de la sédentarité. Un journal est remis à l'élève pour qu'il le renseigne chaque fois qu'il retirera l'accéléromètre. Les seuils de signification de l'activité physique par Accéléromètre: I'OMS considère comme satisfaisant (actif), tout enfant, menant une activité physique de plus de 60 minutes par jour et sédentaire si l'activité physique journalière est inférieure à 60 minutes. La tension artérielle a été mesurée avec un tensiomètre numérique automatique digital fixé à un brassard de taille appropriée sur le bras gauche. Trois mesures de pression artérielle systolique (PAS) et pression artérielle diastolique (PAD) doivent être effectuées et au cours de l'analyse des données, on calculera la moyenne de la deuxième et troisième lecture. Selon le « National High Blood Pressure Education Program », en fonction du sexe, de l'âge et du percentile pour la taille les seuils appliqués sont: pré-hypertension (90ème percentile $\leq$ PAS et/ou PAD < 95ème percentile) et hypertension : PAS et/ou $P A D \geq 95$ ème percentile.

Pour évaluer le niveau de connaissance des élèves en nutrition, la bonne réponse à la question est notée 1 point et la mauvaise réponse zéro. Les enfants ont été scorés comme suit: mauvaise connaissance en nutrition (Score < 4), moyenne connaissance en nutrition (Score 4 -7 ), Bonne connaissance en nutrition (Score $\geq 7$ ).

Pour évaluer l'Attitude par rapport à une alimentation saine, les questions ont été scorées sur 4 points: mauvaise attitude (Score < 2), moyenne attitude (Score 2 - 3) et bonne attitude (Score $\geq 4$ ).

Pour apprécier l'estime de soi, les enfants ont désigné parmi 9 silhouettes classées dans l'ordre de corpulence croissante, les images auxquelles ils ressemblent et aimeraient ressembler. Ils ont été catégorisés comme suit: bonne estime de soi (si l'élève s'identifie à une image et à laquelle il aimerait ressembler), surestimation de soi (si l'élève s'identifie à une image mais aimerait ressembler à une autre image de plus petite corpulence) et sous-estimation de soi (si l'élève s'identifie à une image mais aimerait ressembler à une autre de corpulence plus grande). Dans tous les cas les deux dernières estimations (sur et sous estimations) sont considérées comme une fable estime de soi.
Pour la Pratique de Mode de vie sain, favorable à la santé les questions retenues ont été scorées sur 11 points : mauvaise pratique de mode de vie (Score < 4), moyenne pratique de mode de vie (Score 4 - 7) et Bonne pratique de mode de vie (Score $\geq 7$ ).

Les données ont été saisies sur Epi-data. Toutes les analyses statistiques ont été effectuées en utilisant le logiciel SPSS version 20. Le texte $\mathrm{Khi}^{2}$ de Pearson a été le test statistique, seuil de $80 \%$ avec un niveau de signification si $p \leq 0.005$. Pour l'analyse des facteurs de risque de l'obésité on a eu recourt à la régression logistique, l'obésité étant la variable dépendante.

\section{Résultats}

Après le contrôle de qualité des mesures de la composition corporelle, 92 participants avec un ratio de 0.73 ont été retenus pour l'analyse des données. La moyenne d'âge était de 9,7 ans, l'IMC de $17,74 \mathrm{~kg} / \mathrm{m}^{2}$. Selon la composition corporelle, la moyenne de l'eau corporelle totale (ECT) était de $60,60 \% \pm 7,8$, la masse non grasse (MNG) était de $79,13 \% \pm 10,23$ et la masse grasse (MG) était de $20,86 \% \pm 10$, 2. Le taux d'hypertension artérielle était de $12 \%$ avec comme PAS et PAD, respectivement $105,6 \mathrm{~mm} \mathrm{Hg}$ et $67,0 \mathrm{~mm} \mathrm{Hg}$. Les caractéristiques des participants en fonction du sexe sont indiquées dans le tableau 1. L'évaluation du statut nutritionnel sur la base de la référence de croissance IMC-pour-âge (5-19 ans) de la classification de l'OMS, indique un taux d'obésité de $6 \%$ (1,78\% pour les garçons et $4,22 \%$ pour les filles) contre $26 \%$ (pour les garçons FM> 25\%: $11,9 \%$ et pour les filles FM> 30\%: 14,1\%) selon la composition corporelle (voir figure). Par rapport au comportement, $58,7 \%$ des écoliers n'avaient pas de mode de vie favorable à la santé, $51,1 \%$ avaient un mauvais point de vue à une alimentation saine. Seulement $16,2 \%$ et $15,8 \%$ des enfants avaient respectivement une bonne connaissance des pratiques nutritionnelles et une bonne estime de soi.

Parmi les enfants obèses, 88,9\% étaient des sédentaires, $45,8 \%$ étaient hypertendus, $70 \%$ avaient de mauvais point de vue alimentaire, $87,5 \%$ avaient un faible estime de soi, $25 \%$ avaient un mode vie favorable à la santé, $54,2 \%$ étaient scolarisés dans des établissements privés, $55 \%$ de leurs deux parents pratiquaient des activités génératrices de revenus.

Parmi les facteurs de risque analysés, nos résultats ont montré une relation statistique entre l'hypertension artérielle et l'obésité ( $p=0,006, O R=0,152)$ et l'estime de soi $(O R=1,207, p=0,041)$

Tableau I : Caractéristiques des écoliers en fonction du sexe

\begin{tabular}{|l|c|c|}
\hline Caractéristiques & $\begin{array}{c}\text { Filles } \\
\text { (n=53) }\end{array}$ & $\begin{array}{c}\text { Garçons } \\
\text { (n=39) }\end{array}$ \\
\hline Age $(\mathrm{an})$ & 9,71 & 9,64 \\
\hline Poids $(\mathrm{kg})$ & 35,5359 & 34,8667 \\
\hline Taille $(\mathrm{cm})$ & 140,45489 & 139,90513 \\
\hline Tour de taille $(\mathrm{cm})$ & 63,2332 & 62,6936 \\
\hline BMl $\left(\mathrm{kg} / \mathrm{m}^{2}\right)$ & 17,7391 & 17,4923 \\
\hline
\end{tabular}




\begin{tabular}{|l|c|c|}
\hline ECT kg & 21,2177 & 20,8058 \\
\hline ECT\% & 59,9545 & 61,4768 \\
\hline MNG kg & 27,4064 & 27,1788 \\
\hline MNG\% & 77,88 & 80,37 \\
\hline MG kg & 8,0645 & 7,5345 \\
\hline MG\% & 22,11 & 19,27 \\
\hline $\begin{array}{l}\text { Pression artérielle } \\
\text { systolique (mm Hg) }\end{array}$ & 105,562 & 102,615 \\
\hline $\begin{array}{l}\text { Pression artérielle } \\
\text { diastolique (mm Hg) }\end{array}$ & 67,038 & 64,821 \\
\hline
\end{tabular}

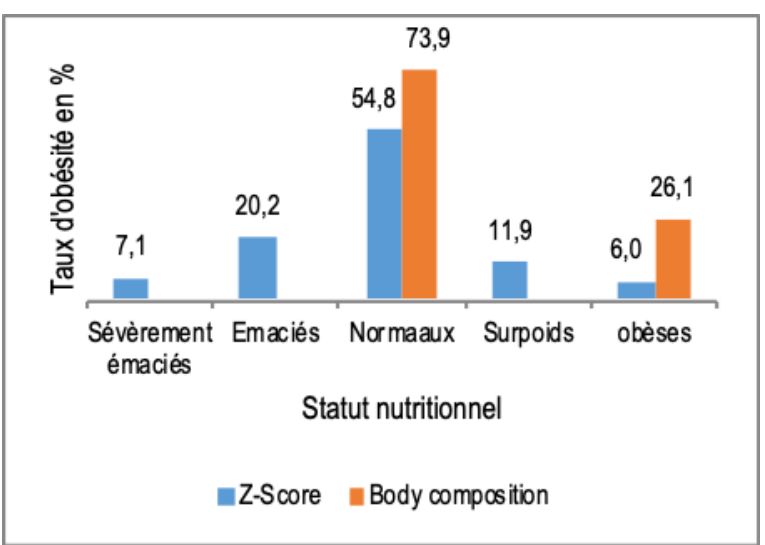

Figure 1 : Fréquence des différentes formes d'obésité selon le Z-score versus la composition corporelles (masse grasse)

\section{Discussion}

De l'obésité découlent la majorité des MNT devenues un problème de santé publique partout dans le monde. Et donc, l'un des enjeux majeurs du 21ème siècle est la réduction de la prévalence de l'obésité. Pour cela, il faut introduire des interventions de changement de comportement alimentaire au sein de la population. Or il est connu qu'il est plus facile d'introduire des interventions au niveau scolaire et que le plus souvent, les habitudes apprises à l'école continuent plus tard dans la vie. Raison pour laquelle la population cible de cette première étude de référence au Mali, a été les écoliers. II s'agissait de déterminer l'obésité infantile par l'évaluation de la composition corporelle et de connaître ses facteurs de risque.

La réalisation de cette étude a porté sur un échantillon de 92 élèves âgés de 8 à 11 ans. La prévalence de l'obésité selon l'IMC était de $6 \%$. Elle était plus élevée chez les filles $4,22 \%$ que chez les garçons $1,78 \%$. Cette prévalence est proche de celles retrouvées en $\mathrm{RCl}$ par K.E. Kramoh et collègues avec $5 \%$, plus fréquente chez les filles $(6,8 \%)$ que chez les garçons $(1,8 \%)$ [9], au Congo 7,1\% par Jean-Robert M. en 2011 [10], au Nigeria par Senbanjo et al. (5,2 \%) en 2007 [11], en Algérie par Mekhancha - Dahel et al. (4 \%) en 2002 [12], Toutefois elle est inférieure à celles signalées au Sénégal par Faye et al. (9,34\%) en 2011 [7] et en Afrique du Sud (13,7\% \%) en 2012 par Monyeki et al. [13]. Par rapport aux pays industrialisés, notre prévalence est très inférieure car dans ces pays, l'obésité est à une vitesse alarmante à cause du niveau de vie très élevé [14]. Cette prévalence était de 18,5\% aux États-Unis en 2016 [15], en France, elle était voisine de $15 \%$ en 2014 [16].

La prévalence de l'obésité selon la masse grasse des élèves par la technique de la dilution isotopique était de $26 \%$. II y'avait plus de filles $14,1 \%$ que de garçons $(11,9 \%)$. Cette différence est aussi observée dans la littérature des pays en voie de développement. Majoritairement les filles sont plus obèses que les garçons car le petit garçon est plus actif dans ces pays que la jeune fille. Dans les pays industrialisés, cette différence entre les sexes est plus prononcée de façon variable, chez le garçon que chez la fille, ce qui a été rapporté par plusieurs études $[9,14]$.

Nos résultats sont similaires à ceux de Diouf et al qui ont aussi rapporté une prévalence d'obésité de 29,1\% par l'évaluation de la composition corporelle versus $8,8 \%$ par I'IMC pour les enfants âgés de 8 à 11 ans [17].

Pour étudier l'implication des différents facteurs de risque de l'obésité, nous avons eu recours à l'analyse de régression logistique linéaire et selon le secteur d'enseignement. Même si la revue littéraire indique que les causes du surpoids et de l'obésité sont multifactorielles [18-20], les résultats de la présente étude ont montré que l'obésité chez les élèves à Bamako n'avait de lien statistiquement significatif avec aucun des facteurs suivants : le statut socioéconomique des parents d'élèves, le type d'école fréquenté, le niveau de connaissance en nutrition, leur comportement par rapport à l'alimentation, la pratique de mode de vie favorable à la santé et la pratique d'activité physique $(p>0.05)$. Certains facteurs comme l'hérédité, le diabète, les apports en macronutriments, le mode de vie moderne, la diminution de l'activité physique, le sédentarisme, le grignotage, la réduction du temps de sommeil ont été clairement identifiés par d'autres chercheurs [20-22].

Dans notre étude, seules la tension artérielle et la faible estime de soi ont eu une relation avec l'obésité infantile. La tension artérielle était significativement $(p=0,006)$ liée à l'obésité. Parmi les enfants obèses, $48,5 \%$ avaient une tension artérielle élevée. Ce résultat est similaire à celui de Giacomo (40\% des adolescents hypertendus sont obèses) [23], qui avait trouvé en 2010 , une relation statistique entre l'IMC et la tension artérielle.

Un lien statistiquement significatif $(p=0,041)$ a aussi été retrouvé entre le niveau d'estime de soi et l'obésité. Près de $87,5 \%$ des élèves obèses avaient une surestimation de leur apparence physique et voulaient ressembler à une autre silhouette, d'où la faible estime de soi. Des études antérieures ont aussi trouvé une relation inverse entre l'obésité durant l'enfance et l'estime de soi [24-26]. Cependant en 2009, par une étude longitudinale, F. 
Wang [27] confirme que la faible estime de soi n'est pas un prédicteur de l'obésité.

Notre étude présente certaines limites. Premièrement, étant donné que notre échantillon était composé de sujets volontaires, il n'était peut-être pas représentatif des écoliers de la tranche d'âge ciblée. En effet l'administration du deutérium par les enfants n'avait pas été appréciée par certains parents d'élèves qui ont refusé leur consentement éclairé. Deuxièmement, après le contrôle de qualité des données, 92 élèves sur 168 furent retenus pour la réalisation des analyses statistiques.

Tout cela peut amener à biaiser la prévalence de l'obésité. Ce qu'il faut retenir dans cette étude c'est que l'excès de graisse corporelle qui est une réalité parmi les écoliers à Bamako ne peut pas être détecté par l'IMC. Par contre, la méthode de l'oxyde du deutérium nous montre qu'une personne apparemment de corpulence normale peut être classé obèse.

\section{Conclusion}

Pour mieux comprendre l'étiologie de l'obésité, dans un premier temps, il est nécessaire de mener d'autres études en améliorant la qualité du dépistage de l'obésité par l'évaluation de la composition corporelle, puis de mettre en place des mesures particulières sur les différents déterminants pour freiner le fardeau de la malnutrition sur le système de santé.

\section{Références}

1. K. E. Djadou, K. Sadzo-Hetsu, K. S. Koffi, E. Tsolenyanu, K. Douti, K. D. Afia, D. Y. Atakouma. Prévalence de l'obésité en milieu scolaire urbain (Togo) Journal de pédiatrie et de puériculture (2010) 23, 335-339,

2. Heidi G Hildreth, Rachel K Johnson, Michael I Goran, and Stephen H Contompasis. Body composition in adults with cerebral palsy by dualenergy X-ray absorptiometry, bioelectrical impedance analysis, and skin fold anthropometry compared with the isotopedilution technique. Am J Clin Nuir 1997 ;66:1436-42 ;

3. Organisation Mondiale de la Santé. Centre des médias obésité et surpoids Aide-mémoire $\mathrm{N}^{\circ}$ 311 Août 2014 www.who.int. Consulté le 15septembre 2017;

4. OMS Principaux repères sur l'obésité et le surpoids https:/xxx.who.int consulté le 29/12/2018 à $10 \mathrm{~h} 40$;

5. Kushner RF, Schoeller DA. Estimate of total body water by bioelectrical impedance analysis. American Journal of Clinical Nutrition 1986: 44; 417-424;

6. Anne Collet. Famine et obésité, la double peine de l'Afrique : 2012 ;

7. Faye J, Diop M, Gati Ouonkoye R, Seck M, Mandengué SH, Mbengue A, et al. Prévalence de l'obésité de l'enfant et de l'adolescent en milieu scolaire à Dakar. Bull Soc Pathol Exot $2011 ; 104: 49-52$;
8. Prévalence de l'obésité statistique mondiale http://www.statistiques-

mondiales.com/obesite.htm;c onsulté le 15 septembre 2017

9. K. E. Kramoh, Y. N.K. N'Goran ? E. AkéTraboulsi, B.C. Boka, D.E. Harding, B.D.J. Koffi, F. Koffi, M.K. Guikahue. Prévalence de l'obésité en milieu scolaire en Côte d'Ivoire. Annales de Cardiologie et d'Angéiologie 61 (2012) 145-149 ;

10. Jean-Robert M. Josiane S. Henry G. M. Aurore M. C. Jean-Louis $N$ Georges $M$. Profil épidémiologique et clinique de l'obésité de l'enfant à Brazzaville (Congo); Cahier de nutrition et de diététique (2011) 46, 259-262 ;

11. Senbanjo 10, Adejuyigbe. Prevalence of overweight and obesity in Nigerian preschool children. Nutrition and health 18 (4) 391-399, 2007 ;

12. Mekhancha-Dahel CC, Mekhancha DE, Bahchachi N, Benatallah L, Nezzal L. Surpoids, obésité : signes de la transition nutritionnelle chez les enfants et les adolescents scolarisés au Khroub, Algérie. Revue d'épidémiologie et de santé publique 53 (5), 569-573, 2005 ;

13. Monyeki A M, Neetens R, Moss S A and Twisk Jos. The relationships between body composition and physical fitness in 14 year old adolescents residing within the Tlokwe local municipality, South Africa: The PAHL study. BMC Public Health 2012, 12 : 374 http//www. Biomedcentral.com/1471-2458/12/374;

14. Dinsa G D, Goryakin $Y$, Fumagalli $E$, and Suhrcke M. Obesity and socioeconomic status in developing countries: a systematic review. doi : 10.1111/j.1467-789X.2012.01017.x ;

15. Craig M. Hales, M. D., Margaret D Carroll, M.S.P.H., Cheryl D. Fryar, M.S.P.H., and Cynthia L. Ogden, Ph, D. NCHS Data Brief. No 288. Octobre 2017 ;

16. Castetbon K. L'évolution récente des prévalences de surpoids et d'obésité chez l'enfant et l'adolescent en France et au niveau international. Archives de pédiatrie 22 (2015) 111-115;

17. Adama D, Theodosia A, Abdel A, Asmaa E H, Noorjehan I J, Cornelia U L and all. Body mass index vs deuterium dilution methode for establishing childhood obesity prevalence, Ghana, Kenya, Mauristius, Morocco, Namibia, Senegal, Tunisia and United republic of Tanzania. Bull World Health organ $2018 ; 96: 772-781 \quad$ doi : http://dx.doi.org/10.2471/BLT.17.205948;

18. Hoelscher DM, Kirk S, Ritchie L, et al. Position of the Academy of Nutrition and Dietetics: interventions for the prevention and treatment of pediatric overweight and obesity. J Acad Nutr 
Diet $2013 ; 113: 1375-95$;

19. Perez-Escamilla R, Kac G. Childhood obesity prevention: life-course framework. Int $\mathrm{J}$ Obes 2013 ; 3 : S3-5 ;

20. N. J. Farpour-lambert, A. Nydegger, S. Kriemler, D. L'Allemand, J. J. Puder. Comment traiter l'obésité de l'enfant? Importance de la prévention primaire. Rev med. Suisse $2008 ; 4$ : 533-6 :

21. Jean Michel Oppert, Marie-Françoise, RollandCachera. Prévalence, évolution dans le temps et conséquences économiques de l'obésité. Médecine/Sciences $1998 ; 14: 939-43$;

22. Reilly JJ, Armstrong, Dorosty AR, et al. Early life risk factors for obésity in childhood: Cohort Study. BMJ 2005 ; $330: 1357$;

23. Giacomo D. Simonetti, Barbara S. Bucher, Monica Ragazzi, Sibylle Tschumi, Mario G. Bianchetti. Hypertension artérielle en pédiatrie. Forum Med Suisse 2010; 10(17) :299-303;

24. S. A. French, M. Story et C. L. Perry. Selfesteem and obesity in children and adolescents, a littérature review. Obesity Research, 3(5), 1995, p.470-490;

25. J. Wardelle et L. Cooke. The impact of obesity on psychological well-being. Best pratice and research Clinical Endocrimology and metabolism, 19(3), 2005. P. 421-440;

26. F. F. Wang et Veugelers. Self-esteem and cognitive development in the era of chilhood obesity epidemic. Obesity Review, 9, 2008, p 615-623;

27. F. Wang, T. C. Wild, W. Kipp, S. Kuhile et P. J ; Veugelers. L'influence de l'obésité infantile sur le développement de l'estime de soi. Travaux de recherche. Statistique Canada, n82-003XPF au catalogue. Rapport sur la santé. Vol 20, $n^{\circ} 2$, juin 2009 . 
Tableau II : Caractéristiques des enfants obèses

\begin{tabular}{|c|c|c|c|c|c|c|c|c|c|}
\hline Age & Poids & Taille & Tt & IMC & ECT & MNG & MG & PAS & PAD \\
\hline $\mathbf{( A n})$ & $\mathbf{( K g )}$ & $(\mathbf{c m})$ & $\mathbf{( c m})$ & $\left(\mathbf{K g} / \mathbf{m}^{2}\right)$ & $(\%)$ & $(\%)$ & $(\%)$ & $(\mathbf{m m ~ H g})$ & $(\mathbf{m m ~ H g})$ \\
\hline 9,83 & 43,565 & 142,248 & 71,815 & 21,067 & 50,054 & 64,779 & 34,660 & 114,13 & 70,158 \\
\hline
\end{tabular}

